\title{
Fabricating nanodiamonds from biomass by direct laser writing under ambient conditions
}

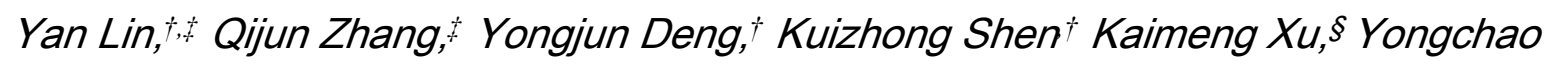
Yu, '† Siqun Wang, ${ }^{*,+}$ and Guigan Fang, ${ }^{*}$

$\dagger$ Institute of Chemical Industry of Forestry Products, CAF; Key Lab of Biomass Energy and Material, Jiangsu Province; Co-Innovation Center of Efficient Processing and Utilization of Forest Resources, Jiangsu Province; National Engineering Lab for Biomass Chemical Utilization; No. 16, $5^{\text {th }}$ Suojin, Xuanwu District, Nanjing 210042, PR China; email address: fangguigan@icifp.cn

* Center for Renewable Carbon, University of Tennessee, 2506 Jacob Drive, Knoxville, TN 37996, USA; email address: swang@utk.edu

\& College of Materials Science and Engineering, Southwest Forestry University, 300 Bailongsi, Panlong District, Kunming, 650224, PR China

$\pitchfork$ Department of Mechanical, Aerospace and Biomedical Engineering, University of Tennessee, 1512 Middle Drive, Knoxville, TN 37996, United States 
Number of pages: 11

Number of Figures: 8

Number of Tables: 3
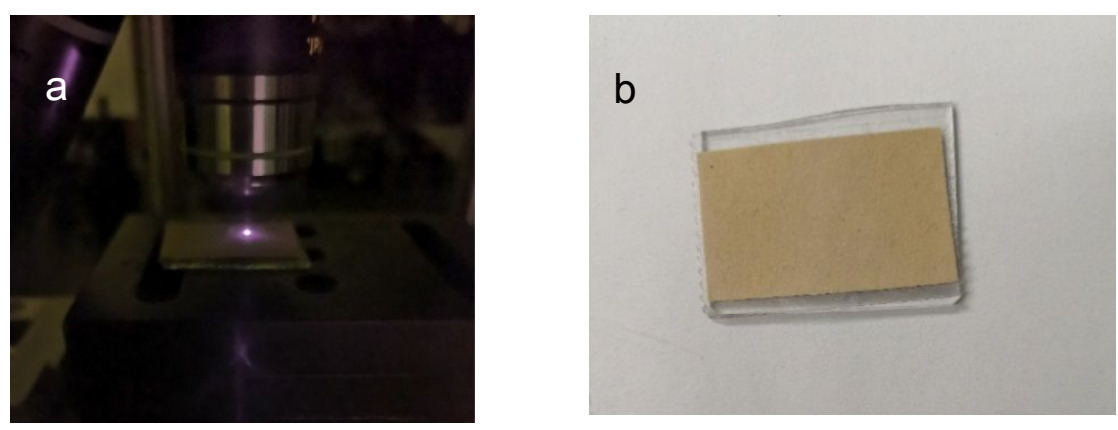

Figure S1. (a) Photograph of laser irradiating the CNF film; (b) the CNF film with paper substrate after laser irradiation.

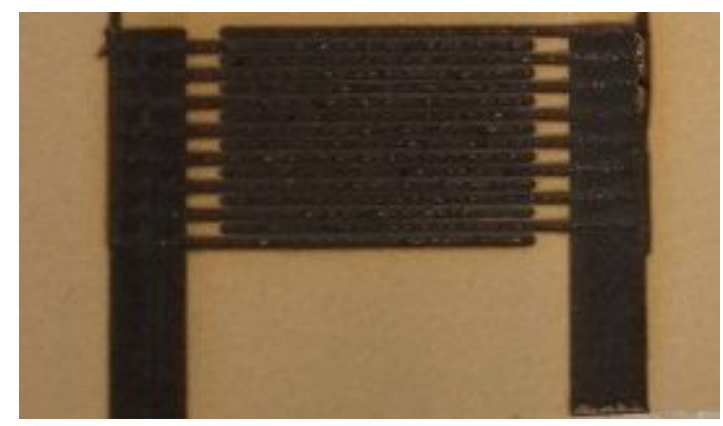

Figure S2. Photograph of a paper-based sensor from LCNF composites by femtosecond laser irradiation. 


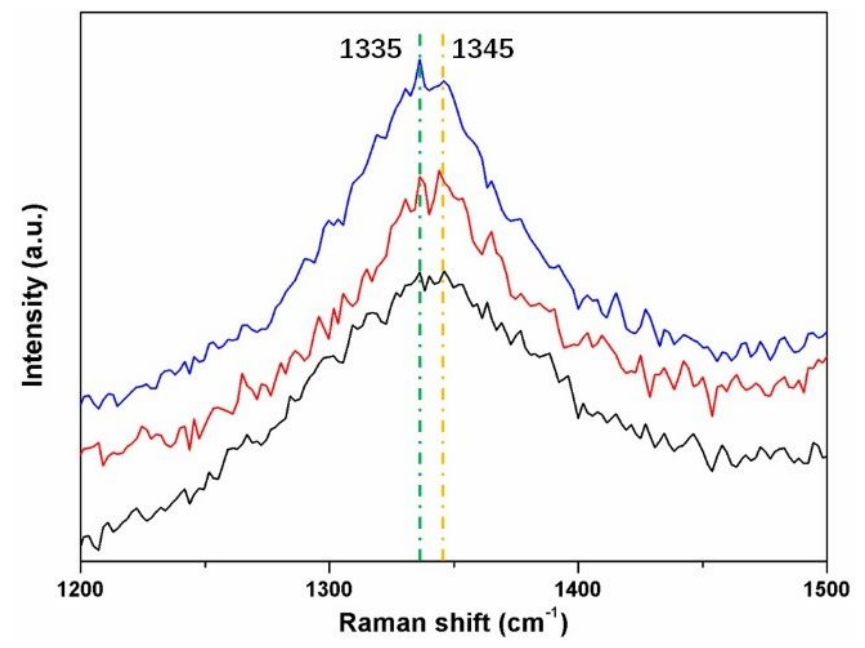

Figure S3. Raman spectra of P600S3.5 pattern. 

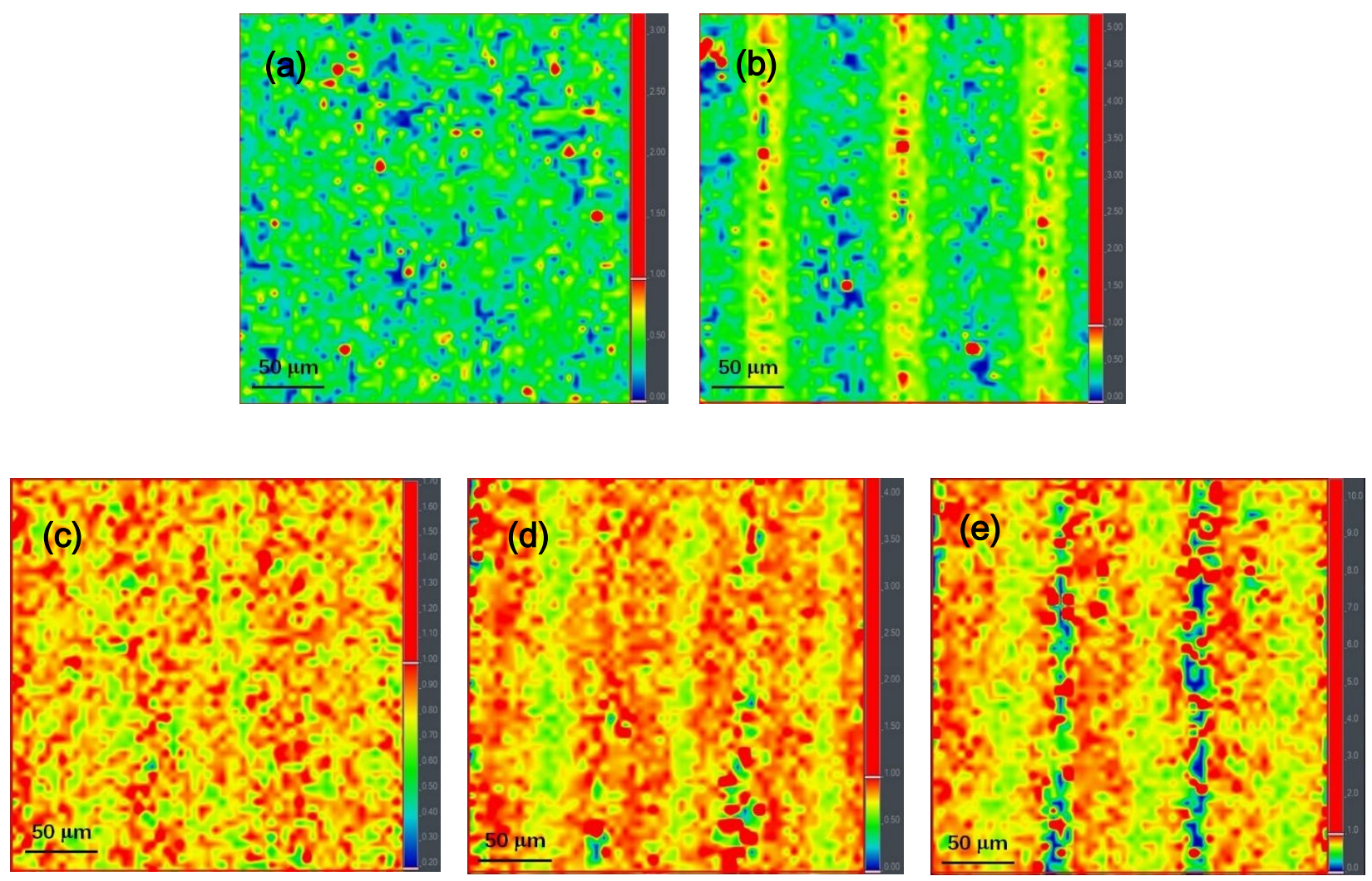

Figure S4. A selection of 2D Raman spectra mapping of $\mathrm{I}_{\mathrm{D}} / \mathrm{I}_{\mathrm{G}}$ : (a) H-LCNF film; (b) P150S3.5 pattern; (c) P300S3.5 pattern; (d) P450S3.5 pattern; (e) P600S3.5 pattern. 

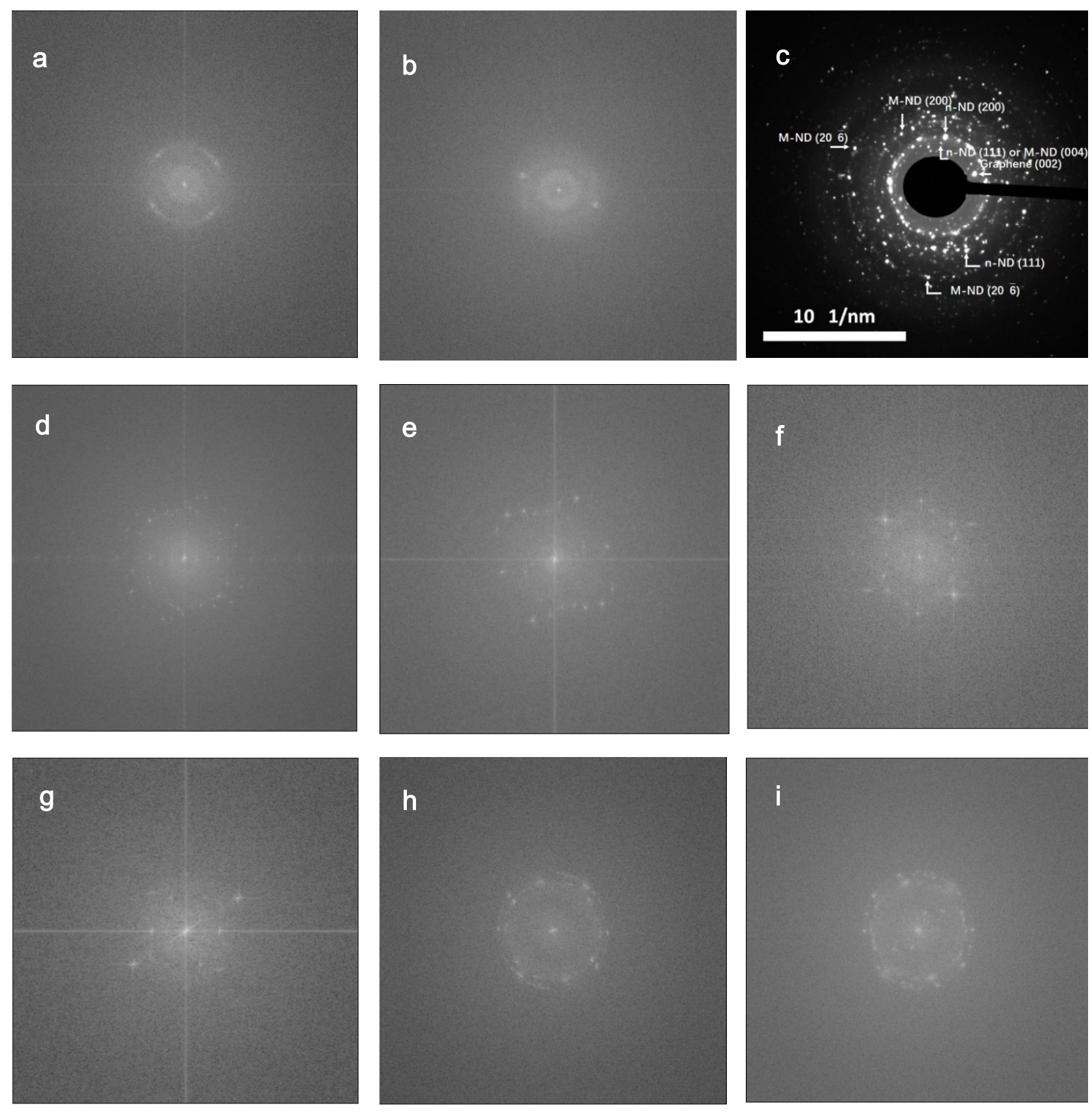

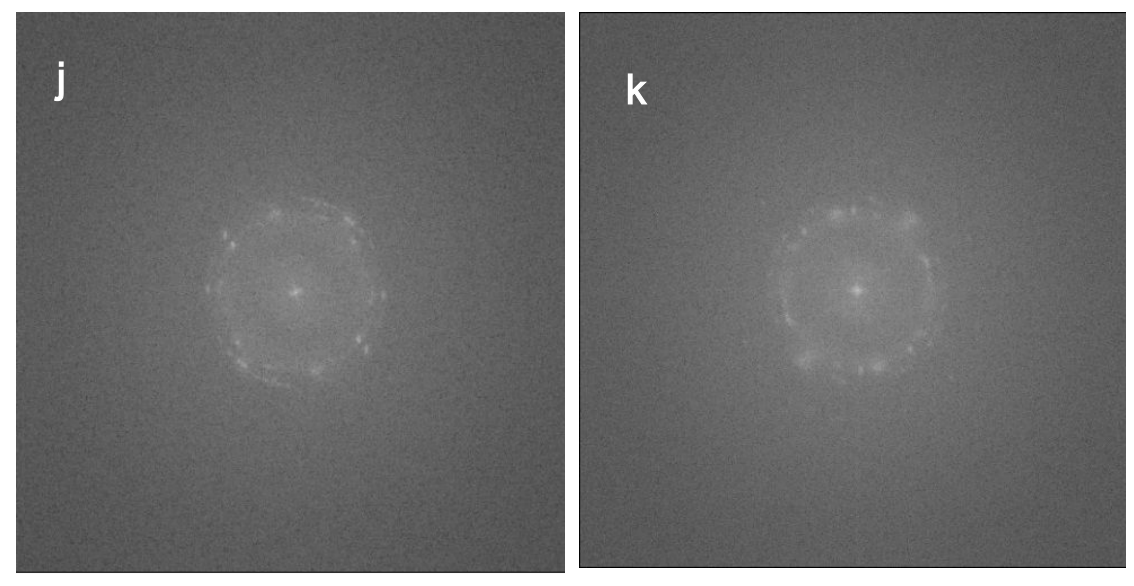

Figure S5. The Fast Fourier Transform (FFT) patterns: (a) the red part in Figure 4a; (b) Figure 4b; (d) upper left part in Figure 4c; (e) upper right part in Figure 4c; (f) lower left part in Figure 4c; (g) lower right part in Figure 4c; (h) Figure 4e; (i) Figure 4f; (j) Figure 4g; (k) Figure 4h. Image (c) was a typical SAED of NDs in P600S3.5 pattern. 

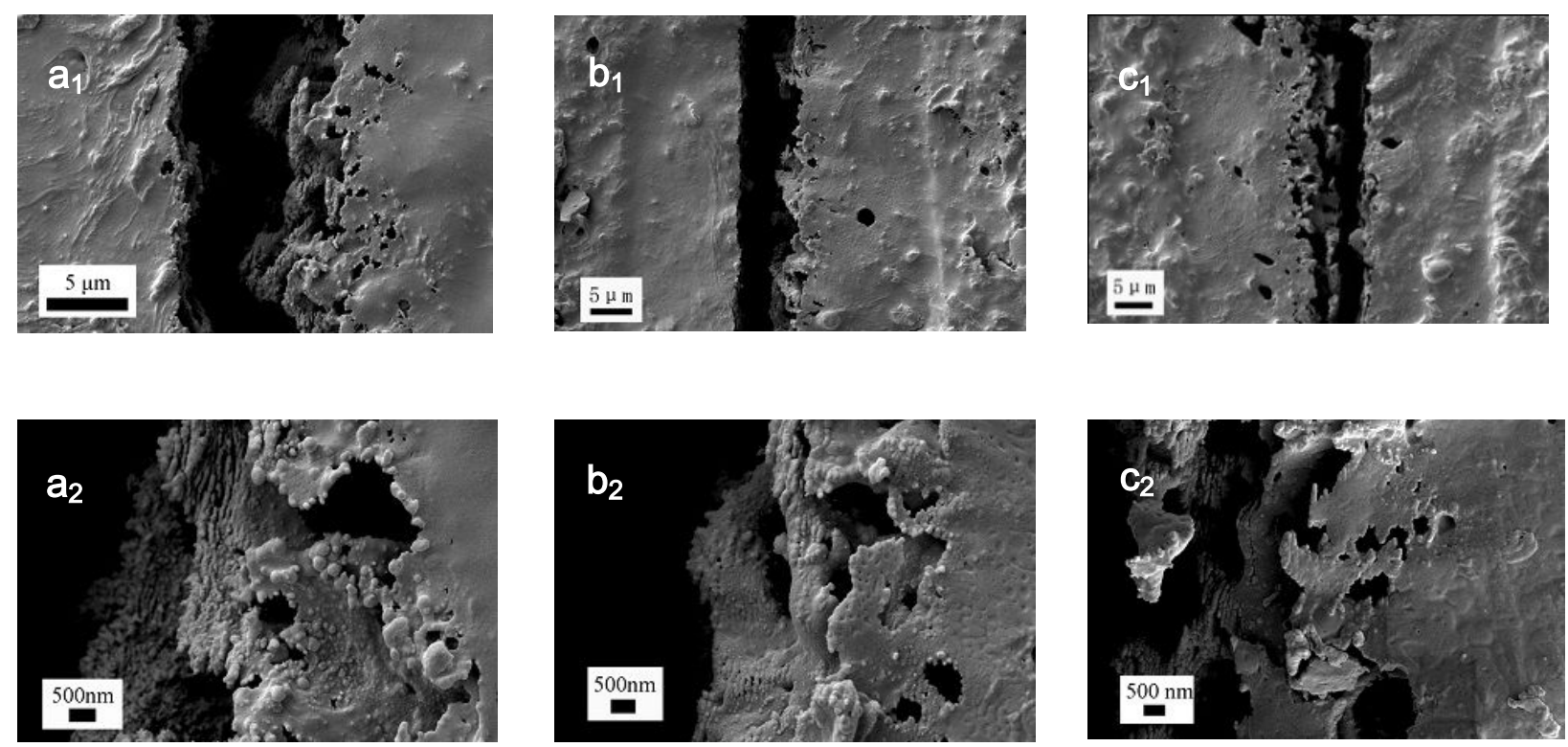

Figure S6. SEM images of laser-induced patterns at different laser writing speeds (H-LCNF

film): (a) $1 \mathrm{~mm} / \mathrm{s}$; (b) $2.5 \mathrm{~mm} / \mathrm{s}$; (c) $3.5 \mathrm{~mm} / \mathrm{s}$.
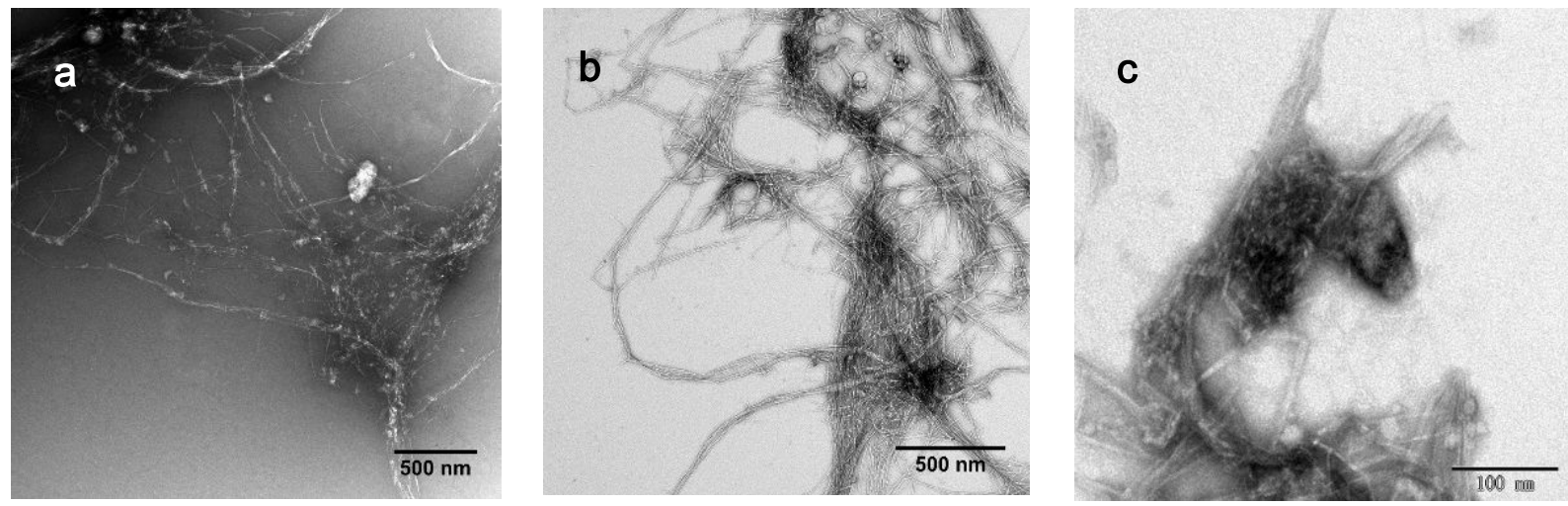

Figure S7. TEM images of LCNF: (a) L-LCNF; (b) M-LCNF; (c) W-LCNF. 

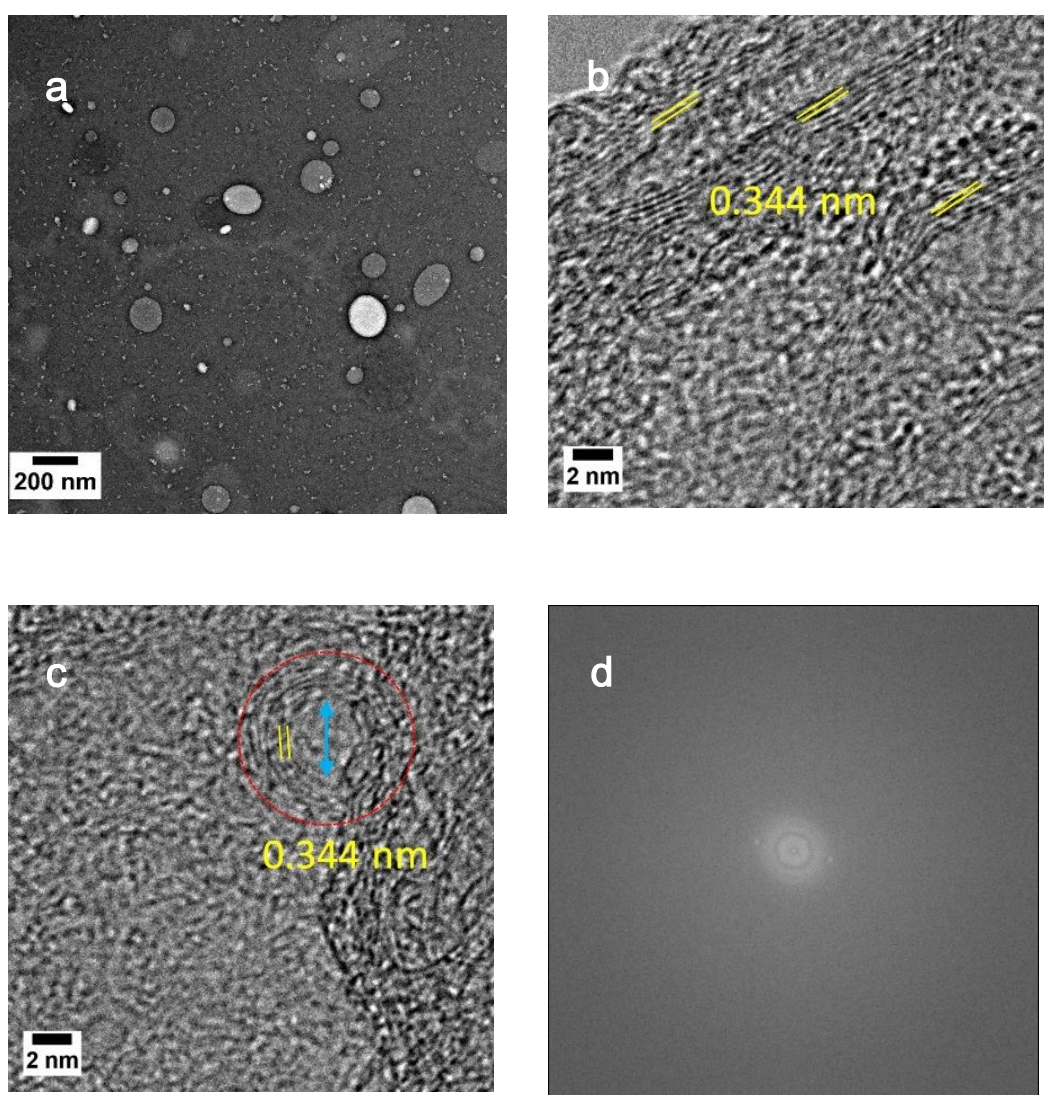

Figure S8. (a) TEM images of nano-lignin; (b-c) High-resolution TEM micrographs of laserinduced nano-lignin pattern in Figure 8d; (d) The Fast Fourier Transform (FFT) patterns of the selected parts in Figure 8f. 
Table S1 Parameters of hydrothermal pretreatment and the main chemical compositions of switchgrass after pretreatment

\begin{tabular}{cccccc}
\hline Groups & Temperature/ & Time/ & Cellulose content/ & Hemicellulose content/ & Lignin content/ \\
& ${ }^{\circ} \mathrm{C}$ & $\min$ & $\%$ & $\%$ & $\%$ \\
\hline Control & - & - & 35.85 & 25.68 & 28.81 \\
H-LCNF & 200 & 60 & 46.19 & 2.48 & 36.67 \\
M-LCNF & 180 & 90 & 47.83 & 2.83 & 30.54 \\
L-LCNF & 180 & 30 & 50.74 & 1.96 & 26.70 \\
W-LCNF & 120 & 60 & 32.91 & 19.33 & 28.66 \\
\hline
\end{tabular}


Table S2 Comparison of d-spacings of laser-induced NDs (LI-ND) with some reported NDs

\begin{tabular}{|c|c|c|c|c|c|c|c|}
\hline Type of ND & $\begin{array}{l}\text { LI-ND } \\
\text { (this study) }\end{array}$ & ${\mathrm{MW}-\mathrm{ND}^{1}}^{1}$ & M-ND ${ }^{2}$ & $n-N^{3}$ & $n-N^{4}$ & h-ND ${ }^{1,5}$ & $\mathrm{ND}^{6}$ \\
\hline \multirow[t]{13}{*}{ d-spacings } & 0.315 & 0.319 & 0.312 & 0.315 & & & \\
\hline & & & $(004)$ & (111) & & & \\
\hline & $0.272-0.273$ & $0.266-0.272$ & & 0.273 & & & \\
\hline & & & & (200) & & & \\
\hline & 0.254 & & & & & 0.253 & \\
\hline & & & & & & (110) & \\
\hline & 0.230 & & & & & & 0.230 \\
\hline & 0.217 & $0.218-0.226$ & 0.217 & & & 0.218 & \\
\hline & & & (200) & & & $(100)$ & \\
\hline & $0.208-0.209$ & 0.209 & & & $0.206-0.209$ & 0.206 & \\
\hline & & & & & (111) & $(002)$ & \\
\hline & 0.154 & 0.153 & 0.151 & & & 0.150 & \\
\hline & & & $(20 \overline{6})$ & & & (102) & \\
\hline
\end{tabular}


Table S3 Square resistance of laser-induced patterns

\begin{tabular}{cccccc}
\hline Groups & P150S3.5 & P300S3.5 & P450S3.5 & P600S3.5 & NL-900 \\
\hline Square resistance $/(\mathrm{k} \Omega / \square)$ & 65.41 & 18.01 & 1.33 & 11.17 & 0.51 \\
\hline
\end{tabular}

\section{References}

(1) Roy, S.; Bajpai, R.; Biro, R. P.; Wagner, H. D. Fast growth of nanodiamond in a microwave oven under atmospheric conditions. J. Mater. Sci. 2020, 55, 535-544.

(2) Huang, Q.; Yu, D.; Xu, B.; Hu, W.; Ma, Y.; YanbinWang; Zhao, Z.; Wen, B.; He, J.; Liu, Z.;

Tian, Y. Nanotwinned diamond with unprecedented hardness and stability. Nature 2014, 510, $250-253$.

(3) Xiao, J.; Li, J. L.; Liu, P.; Yang, G. W. A new phase transformation path from nanodiamond to new-diamond via an intermediate carbon onion. Nanoscale 2014, 6, 15098-15106.

(4) Wen, B.; Zhao, J. J.; Li, T. J. Synthesis and crystal structure of n-diamond. Int. Mater. Rev. 2007, $52(3), 131-151$.

(5) Yang, L.; May, P. W.; Yin, L.; Smith, J. A.; Rosser, K. N. Growth of diamond nanocrystals by pulsed laser ablation of graphite in liquid. Diam. Relat. Mater. 2007, 16, 725-729.

(6) Das, T.; Saikia, B. K. Nanodiamonds produced from low-grade Indian coals. ACS Sustain. Chem. Eng. 2017, 5, 9619-9624. 